\title{
Significance of Rheumatic Myocarditis in the Etiology of Heart Failure in Rheumatic Heart Disease.
}

\section{S. Siew}

\section{Division of Pathology, College of Osteopathic Medicine, Michigan State University, East Lansing, MI 48824}

Rheumatic heart disease has been with us since the dawn of Medicine, yet, it still presents a major challenge to medical science, as there are many unanswered questions, such as the exact relationship between the Group A B Hemolytic streptococcal pharyngitis and the rheumatic process and the cause of heart failure in rheumatic heart disease. The rheumatic lesion in the heart is a pancarditis, involving all three layers: endocardium, parietal and valvular, myocardium and pericardium, as well as the impulse conduction system. At all stages, the valvular affect is the prominent lesion on gross examination, more particularly, in the chronic stage when the valvular deformities are very obvious and give rise to serious hemodynamic sequelae. On that basis, it has been considered that this is the major factor in the development of heart failure in rheumatic heart disease. Recent investigations found that levels of troponin I were not elevated in rheumatic subjects. This indicates that there is no necrosis of muscle fibers in the rheumatic process, and it has been taken as further support for the hypothesis that heart failure is caused by the valvular lesions.

At the light microscopic level, the myocardial lesions are not striking. They tend to be focal, affecting, primarily, the arterioles and the periarteriolar supporting tissue. However, there is extension along the fascial planes. In the acute phase, there are varying degrees of vasculitis and the formation of the pathognomonic cellular reaction, the Aschoff nodule, is in close relationship to the arterioles. This is followed in the chronic phase by fibrosis, resulting in the characteristic concentric, onion skin, periarteriolar lesion. Thus, a barrier is formed between the myofibers and the source of their blood supply. This must lead to a disturbance of the normal homeostasis of the cardiac myofiber, as no part of it should be farther than $2 \mu \mathrm{m}$ from its blood supply. There is evidence of atrophy and foci of vacuolation of the myofibers. It is difficult ot interpret the vacuolation in autopsy material, as it may be due to post mortem degeneration.

Examination at the ultrastructural level of fresh surgically resected left atrial appendages and papillary muscles, during valve replacement has high lighted the lesions of the arterioles and degenerative changes in the muscle fibers. Fig. 1 shows the extreme degree of end-arteritis with profound encroachment upon the lumen of the arteriole. There is obvious widening of the fascial planes between the myofibers, with the presence of collagen and elastic fibers in the supporting tissue, increasing the distance between the myofibers and their blood supply.

There was gross evidence of parenchymatous degeneration of the myofibers. This ranged from crowding of the mitochondria, foci of vacuolation, increase of lipofuscin and the formation of concentric, osmiophilic structures, "myelin figures" within the myofibers (Fig. 2). Histochemistry showed this to be fatty degeneration. Such severe myofiber damage must make a significant contribution to the heart failure in rheumatic heart disease. 

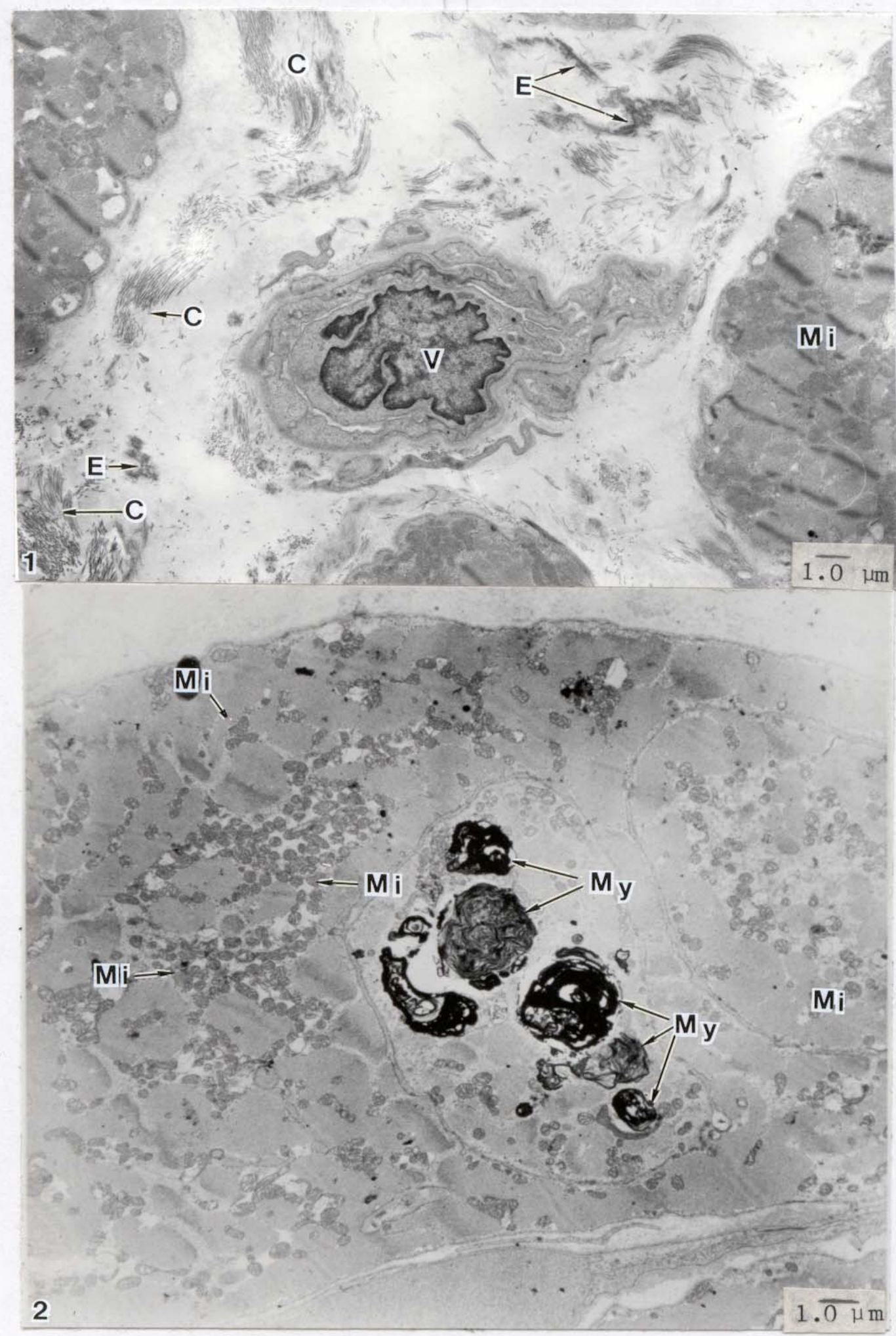

FIG. 1. Endarteritis with severe encroachment upon the lumen - V. Wide separation of myofibers due to increase of interstitial tissue: C-Collagen; E-Elastic fibers. Microwding of mitochondria.

FIG. 2. Myofiber degeneration. My-Myelin figures. Mi - crowding of mitochrondia. 